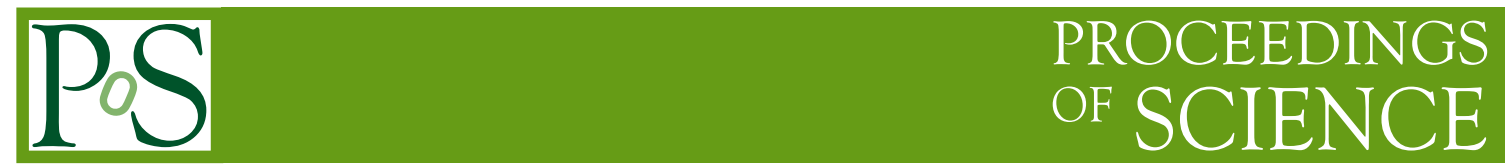

\title{
Atmospheric Neutrinos and Proton Decay
}

\section{Roger A. Wendell*}

Department of Physics, Kyoto University, Kyoto, Japan

E-mail: rawescphys.kyoto-u.ac.jp

While there is as-yet no indication of proton decay, lifetime limits have been extended to new extremes prompting the construction of megaton-scale detectors. At the same time, efforts to understand the main background to such searches, atmospheric neutrinos, have yielded a variety of information on their masses, mixings, and even interactions with matter. This presentation discussed recent experimental results as well as expectations for the next generation of detectors in the study of atmospheric neutrinos and the search for proton decay.

XXIX International Symposium on Lepton Photon Interactions at High Energies - LeptonPhoton2019 August 5-10, 2019

Toronto, Canada

${ }^{*}$ Speaker. 
Early grand unified theories (GUTs) predicted proton lifetimes in the range of $10^{30 \pm 2}$ years and decays into experimentally observable final states such as $e^{+} \pi^{0}$. Since such processes explicitly violate baryon number, they would be expected to play an important role in the evolution of the early universe, fulfilling one of the conditions necessary to explain the current matter-dominated universe observed today. At the same time, GUT models have other attractive features including unification of the strong, weak, and electromagnetic forces at around $10^{16} \mathrm{GeV}$, quantization of electric charge, and, depending upon the gauge symmetry of the model, predictions of right-handed neutrinos, which may be necessary to understand the very light (left-handed) neutrinos (see [1] and references therein for a review). These motivated the construction of experiments in the 1980's, in particular the water Cherenkov Kamiokande and IMB experiments, to search for nucleon decays, a generic prediction of these theories.

Models based on an SU(5) gauge symmetry predicted that those experiments would observe between $O(10) \sim O(100 k)$ nucleon decay events per kiloton per year. At the same time atmospheric neutrinos were known to be a potential background, particularly interactions of the form $v_{e}+p \rightarrow$ $e^{-}+\pi^{0}+n$, which produce the same final state as the signal since those experiments are invisible to neutrons and have no charge sign discrimination. In water these backgrounds are expected to be relatively low, roughly 0.5 interactions per kiloton per year, making the prospects for a discovery straight-forward. By the end of observations neither experiment had observed evidence for nucleon decays, placing limits on $p \rightarrow e^{+} \pi^{0}$ of $2.6 \times 10^{32}$ years [2] (Kamiokande-II) and $5.38 \times 10^{32}$ years [3] (IMB-3), and thereby comfortably ruling out the first generation of GUT models. Studies of the atmospheric neutrino backgrounds at both experiments observed a reduced number of $v_{\mu}$-like interactions than predicted by atmospheric neutrino flux models $[4,5]$.

These results prompted the construction of the then next-generation experiment, Super-Kamiokande, to continue searching for nucleon decay and to additionally study the atmospheric neutrino flux in detail. In 1998 the experiment announced an energy and zenith angle deficit of the $v_{\mu}$ flux with no distortion of the $v_{e}$ flux, providing evidence for neutrino oscillations of the form $v_{\mu} \leftrightarrow v_{\tau}$ and therefore massive neutrinos [6]. This observation led to a proliferation of similar measurements which have shown mixing between all three active neutrino states consistent with two mass differences and three mixing angles as well as hints of more exotic oscillations (see [7] for a summary and review).

Amidst this background the search for nucleon decay as well as the study of atmospheric neutrinos continues today. Importantly, the wide range of energies and path lengths available to the atmospheric neutrinos makes them well-suited to studies of exotic oscillation scenarios as well as open questions in the current Pontecorvo-Maki-Nakagawa-Sakata (PMNS) mixing paradigm, such as the ordering of the neutrino masses and the value of the CP-violating phase. While nucleon decay remains to be seen, increasing exposures as well as plans for next-generation experiments keep hope alive for a discovery but also make it more likely to observe background events. Not only does this motivate a better understanding of the atmospheric flux itself, but also better background rejection techniques. This talk reviews recent results in the study of atmospheric neutrinos and searches for proton decay. 


\section{Atmospheric Neutrinos}

Since the discovery of neutrino oscillations, measurements by accelerator and reactor experiments have determined the parameters of the PMNS mixing framework with a precision that exceeds measurements by atmospheric neutrinos. Though it is now known that the three mixing angles, $\theta_{12}, \theta_{13}$, and $\theta_{23}$, as well as two mass differences, $\Delta m_{21}^{2}$ and $\Delta m_{32}^{2}$, are non-zero, the sign of $\Delta m_{32}^{2}$ (the "mass hierarchy") and whether neutrino oscillations violate CP (the value of $\delta_{C P}$ ) remain unknown. Atmospheric neutrinos have sensitivity to effects induced by both of these parameters. While these can be argued as the main focus of studies with this source over the last few years, several efforts to probe exotic types of oscillations as well as to use them as to study neutrino scattering processes have been advanced as well. Each of the above is made possible by the wide range of energies ( $100 \mathrm{MeV}$ to $100 \mathrm{TeV}$ ) coupled with the large variation in neutrino flight lengths, $O(10) \sim 13,000 \mathrm{~km}$.

To study the neutrino mass hierarchy, experiments rely on asymmetric matter effects on the atmospheric neutrino and antineutrino fluxes. At energies between approximately two and ten $\mathrm{GeV}$ enhanced oscillations are present for core-crossing neutrinos via MSW and parametric resonances if the mass hierarchy is normal $\Delta m_{32}^{2}>0$. In this case antineutrinos experience no such enhancement. Conversely, if the hierarchy is inverted, $\Delta m_{32}^{2}<0$, antineutrino oscillations are enhanced and neutrino oscillations are not. This induces an upward-going excess (deficit) of electron (muon) neutrinos from $v_{\mu} \rightarrow v_{e}$ oscillations for the normal hierarchy and similarly for antineutrinos if the hierarchy is inverted. The influence of $\delta_{C P}$ appears primarily as a modulation of the flux below $1 \mathrm{GeV}$, but also has a sub-leading impact on the size of the matter resonances above.

Super-Kamiokande studies atmospheric neutrinos of both flavors to simultaneously study the mass hierarchy and value of $\delta_{C P}$, though its sensitivity to both is dominantly from its electronneutrino-like samples. Based on a 328 kton-year exposure it has measured the atmospheric mixing parameters as $\sin ^{2} \theta_{23}=0.588_{-0.064}^{+0.031}$ and $\Delta m_{32}^{2}=2.5_{-0.20}^{+0.13} \mathrm{eV}^{2}$ [8]. Here, the preferred hierarchy is normal with a significance of $\mathrm{CL}_{s}=82.9-96.7 \%(91.9-94.4 \%)$ excluding (including) constraints from the T2K experiment. A weak constraint for $\delta_{C P} \sim 3 \pi / 2$, consistent with other measurements was also observed. Similarly, the IceCube collaboration has made a complementary study of the mass hierarchy using 3 years of DeepCore data with an energy threshold of $5 \mathrm{GeV}$ [9]. This study uses both track-like (primarily charged current $v_{\mu}$ interactions) and cascade-like (primarily charged current $v_{e}$ and neutral current interactions) as well as an intermediate sample. Assuming $\sin ^{2} \theta_{23}=0.5$ as indicated by accelerator experiments the expected sensitivity is $\sim 0.5 \sigma$. The data result suggests a weak preference for the normal hierarchy, $\mathrm{CL}_{s}=83.0 \%$. Due to the higher energy threshold, the sensitivity to $\delta_{C P}$ is limited at present.

Below $100 \mathrm{GeV}$ there are no $v_{\tau}$ in the atmospheric neutrino flux, but they are generated at lower energies due to $v_{\mu} \rightarrow v_{\tau}$ oscillations. Searching for their interactions is not only a means of verifying muon neutrino oscillations, but is additionally a means of constraining their interaction cross section and can ultimately be used to test the unitarity of the PMNS mixing matrix. At IceCube oscillation-induced $v_{\tau}$ produce cascade-like events as most interactions proceed via deep inelastic scattering and additional particles are produced in the prompt decay of the $\tau$ itself. Nonetheless the collaboration has extracted a $\tau$-like signal using a multi-stage boosted decision tree selection that disfavors the null hypothesis (no oscillation-induced $v_{\tau}$ ) by $3.2 \sigma$ [10]. The normal- 
ization of the $\tau$ signal is measured to be $0.73_{-0.24}^{+0.30}$, where 1.0 represents the nominal expectation. Importantly this result includes both neutral- and charged-current contributions of the signal. Consistency of the analysis against the standard PMNS oscillation scenario was also checked, yielding $\sin ^{2} \theta_{23}=0.58_{-0.13}^{+0.04}$ and $\Delta m_{32}^{2}=2.55_{-0.11}^{+0.12} \mathrm{eV}^{2}$, both of which are consistent with results from accelerator and other atmospheric neutrino measurements.

Due to the large energies and path lengths covered by atmospheric neutrinos they are additionally a useful probe of exotic oscillation scenarios. Measurements at short-baseline accelerator experiments that cannot currently be reconciled with the standard PMNS framework hint at the possibility of a fourth, sterile neutrino separated from the other neutrino masses by $\Delta m_{41}^{2} \sim 1 \mathrm{eV}^{2}$. Though such a large mass splitting produces an oscillation frequency too fast to be resolved with atmospheric neutrinos, the additional mixing parameters needed to accommodate the sterile state can produce sizable changes in the $v_{\mu} \leftrightarrow v_{\mu}$ survival probability. Whereas ordinary three-neutrino PMNS oscillations are suppressed above $50 \mathrm{GeV}$, the introduction of a sterile neutrino can produce significant $v_{\mu} \rightarrow v_{x}$ transitions up to $\mathrm{O}(100) \mathrm{GeV}$. Using a 10-year exposure of the Antares detector, no significant deviation of the track-like event sample (primarily $v_{\mu}$ interactions was observed indicating no evidence for this scenario. Accordingly, limits on the sterile mixing parameters $\left|U_{\mu 4}\right|<0.007$ and $\left|U_{\tau 4}\right|<0.40$ were obtained [11], both of which are consistent with results from Super-Kamiokande and IceCube.

As a fundamentally interferometric effect, neutrino oscillations can be a highly sensitive probe of small departures from PMNS mixing. Violations of Lorentz invariance, for instance, can thereby produce large oscillation effects as the corresponding operators induce effects with frequencies proportional to $L \times E^{d-3}$, where $d$ is the dimension of the operator. For $d>2$ this necessary produces oscillations that differ greatly from the ordinary $L / E$ predicted by PMNS-style mixing. As in the sterile neutrino case, many of these effects are most visible at very large energies. The IceCube collaboration used a two year exposure of events with a visible muon energy between $400 \mathrm{GeV}$ and $18 \mathrm{TeV}$, to yield the most sensitive constraints on the strength of Lorentz invarianceviolating operators for dimensions $d=3$ to $d=8$ using neutrinos to date. Though too extensive to list here the limits range in the $10^{-24} \mathrm{GeV}(d=3)$ to $10^{-45} \mathrm{GeV}^{-4}(d=8)$ [12].

Characterization of the atmospheric neutrino flux at extremely high energies has become increasingly important with the discovery of $\mathrm{PeV}$ cosmic neutrinos. According the the standard model the neutrino interaction cross section scales linearly with energy, keeping $\sigma / E$ constant until roughly $\mathrm{TeV}$ energies, where the momentum transfer becomes comparable to the weak boson masses and this ratio begins to decrease. This implies that at these energies the flux of neutrinos from below the horizon should decrease due to increasing absorption within the Earth. At the same time additional interactions due to new heavy particles, such as leptoquarks, for example, can augment the cross section and thereby further deplete the atmospheric neutrino flux. In Ref. [13] the IceCube collaboration demonstrated the first neutrino cross section measurement at energies above $370 \mathrm{GeV}$. Using neutrino absorption they measured the cross section to be $1.30_{-0.19}^{+0.21}$ (stat. $)_{-0.43}^{+0.39}$ (syst.) times the standard model expectation. The result is in agreement with the standard model prediction and has established this technique for higher statistics measurements at future facilities. 


\section{Proton Decay}

Though atmospheric neutrino studies have produced many more positive results over the last two decades, searches for proton decay are ongoing. With a better understanding of the neutrino backgrounds many searches are underway to mitigate their impact on nucleon decay studies. Broadly speaking, the two modes, $p \rightarrow e^{+} \pi^{0}$ and $p \rightarrow \bar{v} K^{+}$, attract the most experimental attention as having large branching fractions in many traditional GUT and supersymmetric (SUSY) GUT models, respectively. Though there are many other potential decay modes and measuring the branching fraction to each is the only way to identify the underlying theory in the event that proton decay exists, they are too many to detail here. The following discussion focuses on the "flagship" modes only, though progress in other modes can be found in the literature (Refs [14] and [15] are recent results from Super-Kamiokande, for instance).

Proton decay into $e^{+} \pi^{0}$ is among the most experimentally viable modes since the final state particles are visible to any detector with a low enough energy resolution. This allows for the initial state to be fully reconstructed, enabling strong background rejection. Super-Kamiokande is currently the largest detector capable of observing this decay and searches for two or three showering rings, whose total momentum and invariant mass are consistent with a either a bound proton (from within an oxygen nucleus) or free proton (from a hydrogen atom). The former has a signal efficiency of $21.8 \pm 0.4 \%(7.7 \pm 0.4 \%)$ for true bound (free) decays and an expected background rate of $1.116 \pm 0.132 \mathrm{ev} / \mathrm{Mton} / \mathrm{yr}$. In contrast the selection efficiency for free proton decays is $78.4 \pm 1.4 \%(4.2 \pm 0.2 \%)$ for true free (bound) decays with $0.030 \pm 0.021 \mathrm{ev} / \mathrm{Mton} / \mathrm{yr}$ of backgrounds. Since 2008 the detector has been able to tag neutrons with roughly $20 \%$ efficiency and this search includes a cut requiring zero neutrons in the final state. This reduces the atmospheric neutrino backgrounds by roughly $50 \%$, since they often produce many neutrons, with minimal loss in signal efficiency. Details of the event selection and efficiency are present in [16]. Following a null observation after a 365 kton-yr exposure of the detector, Super-Kamiokande has established a preliminary limit on decays into this channel of $\tau_{p}<1.9 \times 10^{34}$ years (90\% C.L.).

Super-Kamiokande is also leading the search for the SUSY-favored $p \rightarrow \bar{v} K^{+}$. However, unlike the $e^{+} \pi^{0}$ mode the presence of the neutrino in the final state means it is not possible to reconstruct the initial proton. Further, the outgoing $\mathrm{K}^{+}$has only $340 \mathrm{MeV} / \mathrm{c}$ of momentum, which is well below the Cherenkov threshold in water, making it invisible to the detector. The search instead makes use of the $K^{+}$decay products, $v_{\mu} \mu^{+}$(64\% branching fraction) and $\pi^{+} \pi^{0}$. For the former, atmospheric backgrounds would overwhelm the monochramatic $(236 \mathrm{MeV} / \mathrm{c})$ muon so the search looks for $6 \mathrm{MeV}$ photons accompanying the de-excitation of a recoiling oxygen nucleus preceding the muon in time by the kaon decay time. In contrast the latter can be nearly completely reconstructed, although the $\pi^{+}$is only slightly above Cherenkov threshold. The signal efficiencies are $9.1 \pm 0.1 \%$ and $10.0 \pm 0.1 \%$, while the background rates are $1.5 \pm 0.3 \mathrm{ev} / \mathrm{Mton} / \mathrm{yr}$ and $2.0 \pm 0.3 \mathrm{ev} / \mathrm{Mton} / \mathrm{yr}$, for the two searches, respectively [17]. A preliminary limit of $\tau_{p}<$ $0.8 \times 10^{34}$ years ( $90 \%$ C.L.) has been set following a $365 \mathrm{kton}$-yr exposure of the detector.

Liquid scintillator detectors have much lower energy thresholds and are therefore capable of directly observing the $K^{+}$from this decay. The challenge for these detectors is disentangling the kaon signal from its decay products when the scintillator time constant is on the same order of the kaon lifetime, $O(10)$ ns. KamLAND is the first large scintillator detector to demonstrate the 
ability to search for these decays using PMT waveform data to identify the signal. Their search obtained a $44.4 \pm 5.3 \%$ signal efficiency with a background rate of $101 \pm 22 \mathrm{ev/Mton/yr} \mathrm{[18].} \mathrm{No}$ candidates were found in an $8.97 \mathrm{kton}-\mathrm{yr}$ exposure of the detector, resulting in a limit of $\tau_{p}<$ $5.4 \times 10^{32}$ years $(90 \%$ C.L.). Though not competitive with the Super-Kamiokande limit, this is the first demonstration of this search method in liquid scintillator and is therefore an important proof-of-principle for future measurements at JUNO [19].

\section{Future Prospects and Conclusions}

Though considerable progress has been made in the understanding of atmospheric neutrinos and their interactions, there remain many unknowns in the now-standard PMNS framework as well as potential discoveries in more exotic scenarios. At the same time, the appeal of proton decay has not diminished despite the lack of experimental indications that the process exists. Partial lifetime limits in the range of $10^{34}$ years are motivating megaton-scale experiments, such as HyperKamiokande and DUNE, to extend the search into the next decade. However, with such large machines the presence of atmospheric neutrino backgrounds will become even more serious and new attempts to mitigate and understand those backgrounds will be needed. At present HyperKamiokande anticipates a 186 kton fiducial volume, roughly 8 times that of Super-Kamiokande, and improved photosensors producing roughly twice the photon yield of the current generation. As a result it expects to observe proton decay at $3 \sigma$ if the proton lifetime is less than $10^{35}$ years for the $e^{+} \pi^{0}$ mode and $3 \times 10^{34}$ years for $\bar{v} K^{+}[20]$.

Future searches for $p \rightarrow \bar{v} K^{+}$will see competition among DUNE, Hyper-Kamiokande, and JUNO. DUNE with $40 \mathrm{kton}$ of liquid argon is expected to have exquisite tracking abilities and is projecting a signal efficiency of $97 \%$ with only $1 \mathrm{ev} / \mathrm{Mton} / \mathrm{yr}$ of background [21, 22] Despite its relatively small volume it is expected to have a $3 \sigma$ discovery potential for a proton lifetime twice as long as that for Hyper-Kamiokande. JUNO will be even smaller at $20 \mathrm{kton}$, but with $65 \%$ efficiency, roughly twice the background rate as DUNE, and a start date in the next few years, and may therefore have the first chance at a new discovery [19]. If the proton lifetime is at the current limit from Super-Kamiokande, JUNO can observe this process within six years of operations.

Atmospheric neutrino physics is expected to continue in each of the above detectors, but as Hyper-Kamiokande will be the largest by far it will have the best sensitivity among them. That being said, its mass hierarchy sensitivity using atmospheric neutrino information alone is only expected to reach $3 \sigma$ after roughly ten years of operations. With higher density photosensor arrays the next-generation neutrino telescopes IceCube-Gen2 (PINGU) [23] and KM3NeT [24] will also address the neutrino mass hierarchy and perhaps before Hyper-Kamiokande or DUNE are constructed. Interestingly, because of their extremely large size both detectors will have the ability to study the tomography of the Earth by measuring the electron density at its core using matter effects in atmospheric neutrino oscillations (see [25] and [26]). These will be the first such measurements and are at present the only proposed method for studying the chemistry of the Earth's core.

Accordingly, the landscape of present and future measurements of atmospheric neutrinos and proton decay is both vast and there is a large potential for new discoveries. 


\section{Acknowledgments}

The author wishes to thank the organizers of Lepton Photon 2019 for their invitation to present the results discussed above and for their successful efforts in making the conference productive and enjoyable.

\section{References}

[1] K. S. Babu et al., arXiv:1311.5285 [hep-ph].

[2] K. S. Hirata et al. [Kamiokande-II Collaboration], Phys. Lett. B 220, 308 (1989). doi:10.1016/0370-2693(89)90058-0

[3] C. McGrew et al., Phys. Rev. D 59, 052004 (1999). doi:10.1103/PhysRevD.59.052004

[4] D. Casper et al., Phys. Rev. Lett. 66, 2561 (1991). doi:10.1103/PhysRevLett.66.2561

[5] R. Becker-Szendy et al., Phys. Rev. D 46, 3720 (1992). doi:10.1103/PhysRevD.46.3720

[6] Y. Fukuda et al. [Super-Kamiokande Collaboration], Phys. Rev. Lett. 81, 1562 (1998) doi:10.1103/PhysRevLett.81.1562 [hep-ex/9807003].

[7] M. Tanabashi et al. [Particle Data Group], Phys. Rev. D 98, no. 3, 030001 (2018). doi:10.1103/PhysRevD.98.030001

[8] K. Abe et al. [Super-Kamiokande Collaboration], Phys. Rev. D 97, no. 7, 072001 (2018) doi:10.1103/PhysRevD.97.072001 [arXiv:1710.09126 [hep-ex]].

[9] M. G. Aartsen et al. [IceCube Collaboration], arXiv:1902.07771 [hep-ex].

[10] M. G. Aartsen et al. [IceCube Collaboration], Phys. Rev. D 99, no. 3, 032007 (2019) doi:10.1103/PhysRevD.99.032007 [arXiv:1901.05366 [hep-ex]].

[11] A. Albert et al. [ANTARES Collaboration], JHEP 1906, 113 (2019) doi:10.1007/JHEP06(2019)113 [arXiv: 1812.08650 [hep-ex]].

[12] M. G. Aartsen et al. [IceCube Collaboration], Nature Phys. 14, no. 9, 961 (2018) doi:10.1038/s41567-018-0172-2 [arXiv:1709.03434 [hep-ex]].

[13] M. G. Aartsen et al. [IceCube Collaboration], Nature 551, 596 (2017) doi:10.1038/nature24459 [arXiv:1711.08119 [hep-ex]].

[14] S. Sussman et al. [Super-Kamiokande Collaboration], arXiv:1811.12430 [hep-ex].

[15] K. Abe et al. [Super-Kamiokande Collaboration], Phys. Rev. D 96, no. 1, 012003 (2017) doi:10.1103/PhysRevD.96.012003 [arXiv:1705.07221 [hep-ex]].

[16] K. Abe et al. [Super-Kamiokande Collaboration], Phys. Rev. D 95, no. 1, 012004 (2017) doi:10.1103/PhysRevD.95.012004 [arXiv:1610.03597 [hep-ex]].

[17] K. Abe et al. [Super-Kamiokande Collaboration], Phys. Rev. D 90, no. 7, 072005 (2014) doi:10.1103/PhysRevD.90.072005 [arXiv:1408.1195 [hep-ex]].

[18] K. Asakura et al. [KamLAND Collaboration], Phys. Rev. D 92, no. 5, 052006 (2015) doi:10.1103/PhysRevD.92.052006 [arXiv:1505.03612 [hep-ex]].

[19] F. An et al. [JUNO Collaboration], J. Phys. G 43, no. 3, 030401 (2016) doi:10.1088/0954-3899/43/3/030401 [arXiv:1507.05613 [physics.ins-det]]. 
[20] K. Abe et al. [Hyper-Kamiokande Collaboration], arXiv:1805.04163 [physics.ins-det].

[21] V. A. Kudryavtsev [DUNE Collaboration], J. Phys. Conf. Ser. 718, no. 6, 062032 (2016) doi:10.1088/1742-6596/718/6/062032 [arXiv:1601.03496 [physics.ins-det]].

[22] R. Acciarri et al. [DUNE Collaboration], arXiv:1601.05471 [physics.ins-det].

[23] M. G. Aartsen et al. [IceCube Collaboration], J. Phys. G 44, no. 5, 054006 (2017) doi:10.1088/1361-6471/44/5/054006 [arXiv:1607.02671 [hep-ex]].

[24] R. Le Breton [KM3NeT Collaboration], Nucl. Instrum. Meth. A 936, 204 (2019). doi:10.1016/j.nima.2018.10.103

[25] S. Bourret et al. [KM3NeT Collaboration], EPJ Web Conf. 207, 04008 (2019). doi:10.1051/epjconf/201920704008

[26] C. Rott, A. Taketa and D. Bose, Sci. Rep. 5, 15225 (2015) doi:10.1038/srep15225 [arXiv:1502.04930 [physics.geo-ph]]. 